\title{
Miranda
}

Revue pluridisciplinaire du monde anglophone /

Multidisciplinary peer-reviewed journal on the English-

speaking world

\author{
16 | 2018 \\ L'expérimental dans la littérature et les arts \\ contemporains
}

\section{Pratiques de l'artboratoire : artistes en résidence à SymbioticA (Perth, Australia)}

\section{Valérie Morisson}

\section{(2) OpenEdition}

\section{Journals}

Édition électronique

URL : http://journals.openedition.org/miranda/11324

DOI : 10.4000/miranda. 11324

ISSN : 2108-6559

\section{Éditeur}

Université Toulouse - Jean Jaurès

Référence électronique

Valérie Morisson, «Pratiques de l'artboratoire : artistes en résidence à SymbioticA (Perth, Australia) », Miranda [En ligne], 16 | 2018, mis en ligne le 31 mai 2018, consulté le 16 février 2021. URL : http:// journals.openedition.org/miranda/11324; DOI : https://doi.org/10.4000/miranda.11324

Ce document a été généré automatiquement le 16 février 2021.

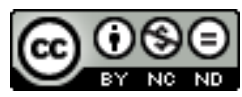

Miranda is licensed under a Creative Commons Attribution-NonCommercial-NoDerivatives 4.0 International License. 


\title{
Pratiques de l'artboratoire : artistes en résidence à SymbioticA (Perth, Australia)
}

\author{
Valérie Morisson
}

\section{Introduction}

1 SymbioticA, école et institut scientifique rattachés à la University of Western Australia située à Perth, est une structure pionnière dans le domaine du bioart. Inspiré du Tissue Culture \& Art Project initié en 1996, cet institut de recherche artistique, qui met laboratoires, technologies de pointe et connaissances scientifiques à la disposition d'artistes en résidence, fut créé il y a 15 ans par les neuroscientifiques Miranda Grounds et Stuart Bunt, ainsi que par Oron Catts, artiste pionnier du bioart. Ce sont à la fois des outils et instruments, des matériaux et des processus expérimentaux que les artistes s'approprient lors de leur résidence. La collaboration durable permise par la résidence est la condition d'une appropriation des techniques scientifiques, d'une réelle compréhension des enjeux éthiques mais également du déploiement de l'expérimentation artistique dans le temps. Les projets ont pour objectif de sensibiliser le public aux questions éthiques afférentes à la recherche scientifique et médicale et aux biotechnologies (l'instrumentalisation du vivant dans l'expérimentation animale, les xéno-transplantations ou l'ingénierie tissulaire par exemple) ${ }^{1}$. Comme l'explique Robert Zwinjenberg: "This artform rather needs constant reflection on the ethical dimensions and paradoxical nature of the specific methods and location of production » (Zwinjenberg 12).

2 Jamais l'univers scientifique n'est simplement reconstitué par les artistes : le travail aboutit à des reconfigurations ou réagencements à l'aune d'un imaginaire lui-même réévalué lors de la confrontation aux potentialités nouvelles offertes par la science. En effet, l'art qui naît de ces croisements disciplinaires et techniques est, comme l'affirme l'artiste Marta De Menezes en résidence à SymbioticA de 2004 à 2005, « une nouvelle 
forme d'art: un art créé dans des tubes à essai et qui utilise les laboratoires comme ateliers » (Voisin). Ces artistes sont engagés dans des recherches sur le vivant, et à ce titre, "They are intentionally transgressing procedures of representation and metaphor, going beyond them to manipulate life itself.» (Catts et Zurr 2004, 167) La transgression à laquelle ils se livrent est double : éthique et esthétique. Oron Catts encourage des pratiques artistiques dans lesquelles le vivant est manipulé, tout en reconnaissant l'existence de dilemmes éthiques ${ }^{2}$ :

As artists, we hope to have a different "contract" with society-we ought to provoke, question and reveal hypocrisies through different tactics: whether through aesthetic, absurd or subtle confrontations. Allowing loss of control or "engineering futility"; making our audience uneasy is an outcome of our own discomfort. All we propose to offer are contestable future scenarios different from the cannon of the contemporary trajectories. (Catts, 2012, 6)

Il est important de préciser que les artistes créent dans un cadre légal garanti par l'institution scientifique et doivent, comme les galeries qui les exposent, obtenir d'un comité d'éthique le droit d'utiliser des tissus humains.

Notre réflexion ne portera pas sur ces dilemmes éthiques maintes fois abordés ${ }^{3}$ mais se penchera sur les transferts et glissements à l'œuvre moins fréquemment évoqués. Au cours de leurs résidences, les artistes travaillant à SymbioticA investiguent des formes radicalement nouvelles, menant l'art à certaines limites ${ }^{4}$. Le bio-art est en effet un art expérimental, au sens où Allan Kaprow l'entend. Ce dernier introduit une distinction fondamentale entre l'art qu'il nomme developmental - que l'on pourrait traduire par progressiste - et l'art expérimental ${ }^{5}$. L'artiste qui expérimente échappe à l'histoire de l'art et aux styles ou manières, y compris celles des avant-gardes; ses préoccupations sont moins formelles que philosophiques; son projet inclut une imprédictibilité qui déplace l'art dans le champ de la vie. Il convient de rappeler qu'étymologiquement ce qui est expérimental est ce qui dépasse les limites, c'est-à-dire les périmètres définis. L'expérimental a donc à voir avec la liminalité. Ainsi, le bio-art, qui rend absolument poreuses les frontières entre l'art et la vie, la métaphore et le réel, induit des formes d'hybridation, «de mélanges des médiums et des territoires symboliques » (Zerbib 45). Il relève d'une démarche expérimentale en ce qu'il impose «la nécessité de reconstruire un univers, un matériau, les perceptions du spectateur ou bien le corps même de l'artiste afin de développer une expérience sensible» (Jeanpierre 310). Ce sont ces liminalités multiples que nous tenterons d'explorer, les interactions ou intersections entre les champs artistique et scientifique, les transferts techniques. Nous retracerons successivement plusieurs dérives nous amenant de la modélisation scientifique au motif artistique, c'est-à-dire de l'imagerie à l'imaginaire; de l'hybridité biologique au chimérique; du protocole au rituel.

\section{De la modélisation scientifique au motif artistique : traces, tracés et inscriptions plurielles}

5 Dans la très célèbre toile de Joseph Wright of Derby, A Philosopher Lecturing on the Orrery, de 1766, les courbes du mécanisme schématisant le système planétaire sont un objet de fascination. Le peintre place le dispositif scientifique et ses courbes au centre de la toile de sorte que la modélisation scientifique devient un modèle artistique. Comme le note Louise Poissant, les sciences de la vie ont de plus en plus recours à des procédés de visualisation, qui sont des outils d'investigation et de compréhension. Plusieurs artistes 
en résidence à SymbioticA ont travaillé à partir de modélisations scientifiques et réfléchi à la question de l'inscription, de la trace ou de l'empreinte. Par ailleurs, tout comme Joseph Wright of Derby avait réintroduit le corps et les émotions dans sa composition en plaçant des personnages ébahis autour du dispositif scientifique, les bio-artistes s'attachent à réincarner les représentations scientifiques. Jennifer Willet, artiste et spécialiste du bio-art, rappelle que ce dernier entend replacer le corps au centre de notre appréhension des biotechnologies trop souvent perçues comme désincarnées (Willet, 2006, 7). Inscrivant sa pensée dans le sillage de celle d'Elizabeth Grosz ${ }^{6}$, elle propose de considérer un corps étendu, non anthropocentrique, pluriel et multiple, qui inclurait différents corps : humains ou non-humains, animés ou nonanimés. En mettant en œuvre des méthodologies incarnées, en replaçant le corps charnel, viscéral, sensitif au cœur de la science, le bio-art déconstruit de multiples hiérarchies: celle qui place l'homme au-dessus des autres organismes vivants mais aussi celle que Platon instaure entre l'empreinte et l'image, entre la vérité et l'imagination. Travaillant à partir d'enregistrements ou de schémas scientifiques, plusieurs artistes transforment le tracé indiciaire en traces, celle du geste de l'artiste mais aussi la trace corporelle ou bien encore la trace mémorielle.

6 Si aux $18^{\mathrm{e}}$ et $19^{\mathrm{e}}$ siècles l'apparition de nouveaux instruments optiques avait généré une fascination pour les questions d'échelles de perception et de représentation, l'emploi d'outils optiques performants permet à certains artistes contemporains de découvrir des images du monde microbien ou bactérien qu'ils traduisent en motifs artistiques et qu'ils re-corporalisent. C'est en effet une exploration endoscopique du corps qu'Adam Zaretsky (l'un des premiers artistes en résidence à SymbioticA) et Tanja Visosevic proposent dans Glack, une vidéo de 19 minutes créée en 2002 grâce à une caméra endoscopique ${ }^{7}$. Les images tantôt floues tantôt extrêmement nettes qui documentent la plongée dans le corps viscéral et visqueux sont accompagnées d'une bande-son constituée de bruits inarticulés, bestiaux ou synthétiques, ou bien encore de musique. Les couleurs saturées qui mettent en valeur certains organes, les variations de points de vue et de vitesse de prise de vue ainsi que les rythmes modulés transforment l'endoscopie en voyage mystérieux au sein d'un univers paysagé. L'imagerie médicale, relevant de l'empreinte telle que la conçoit Platon, c'est-à-dire une trace fidèle approchant la vérité, est ici transformée en matériau artistique fait de couleurs, formes, textures variées et propres à susciter des émotions telles que le dégoût. Les organes mous et informes évoquent un corps répugnant, trace psychique collective du corps archaïque primaire et abject.

7 Lors de sa résidence à SymbioticA et dans le cadre d'un projet à visée écologique intitulé Adaptation (2008-2010), l'artiste australienne Carmel Wallace a également utilisé des modes de capture scientifiques qu'elle a transformés en motifs artistiques. Son travail, centré sur l'écosystème du lac Clifton, au sud-ouest de l'Australie, et ses stromatolithes (les plus anciens organismes vivants bio-construits par des communautés bactériennes), fut encadré par Guy Ben-Ary, spécialiste de l'imagerie microscopique, et par la paléontologue et géologue Kathleen Grey, qui travaille sur la structure interne des stromatolithes. L'artiste a pu mettre au point, de manière expérimentale, un mode de saisie des vues microscopiques à la croisée de l'artistique et du scientifique :

My usual repertoire of tools of capture-camera, pencil, ink, paint, found materials

-were reprioritised as I honed in on the microscopic detail of my subject-initially

the water and organisms surrounding the thrombolites of Lake Clifton, followed by 
the surface and internal structure of a particular thrombolite sample. Whilst attempting to visualise a journey into the past, from the outer layers to the centre of this life form, my investigation encompassed the aesthetics of microscopic detail as I explored ways of transferring knowledge gained scientifically into my visual vocabulary and artworks.$^{8}$

Son œuvre, constituée d'une série de photographies et d'un film court, offre un point de vue panoramique d'abord, puis microscopique ensuite, sur cet écosystème. Composition artistique et composition biologique s'entremêlent. Certaines photographies montrant la structure fondamentale des gouttes d'eau évoquent les œuvres abstraites précédemment créées par Wallace: "the water droplets became living organisms, fascinating worlds opening up numberless possibilities of compositions and shapes.» (Wallace 14) Conçu à partir d'une échelle microscopique, ce travail établit un parallèle entre la trace fossilifère et la gravure : à la surface de l'image les multiples traits et motifs enregistrés mais travaillés par l'artiste évoquent les incisions du graveur. La dissolution des couleurs et les effets de transparence sont quant à eux proches de l'aquarelle. Ce qui était trace, c'est-à-dire signe ou symptôme à déchiffrer d'une présence passée, devient tracé, geste, inscription.

Les mêmes notions s'avèrent centrales dans Proto-Animate 20, installation conçue en 2001 par Andre Brodyk avec l'aide d'Oron Catts. L'artiste utilise la technique de modification génétique pour réaliser des portraits rappelant les effigies romaines. Il nomme cette technique Genetically Modified Painting, expression croisant les champs artistiques et scientifiques. Les portraits organiques sont présentés au sein d'incubateurs ressemblant à des cadres placés sur d'anciens pupitres en bois dans une salle de classe reconstituée. L'œuvre aborde la question de la trace, de la mémoire et de l'oubli. L'obscurité, les pupitres et le tableau noir où sont notées des formules d'ADN intronique partiellement effacées plongent le visiteur dans un passé intime. L'idée selon laquelle l'ADN est ce qui fonde biologiquement notre singularité dialogue avec la tradition artistique du portrait dont la vocation est de saisir l'individualité du modèle. Pour réaliser ses peintures génétiquement modifiées, Brodyk utilise une partie d'ADN associée à la maladie d'Alzheimer qu'il réactive en introduisant une bactérie. Il s'agit plus précisément d'introns, des éléments de séquences non codantes de l'ADN. Nés d'une contamination active, les portraits disparaissent peu à peu. L'idée du protoanimé (des introns qui demeurent inactifs et latents mais qui peuvent être activés) fascine l'artiste, préoccupé par la question de l'oubli. Chaque portrait, résultat d'une contamination par des organismes vivants, est singulier et en constante mutation. Les introns, qui étaient pour les scientifiques porteurs de signes ou symptômes, deviennent une matière artistique générant l'inscription de la singularité profonde mais mutante de chacun.

Lorsque des techniques scientifiques d'enregistrement donnent lieu à des tracés, lorsque les modélisations ou schématisations reposant sur des signes deviennent des traces plurielles, l'imagerie scientifique fait image. C'est dès lors l'imaginaire qui est réintroduit. L'image, eikõn, est exclue du domaine des sciences qui tend à rationaliser le réel à partir de conceptualisations et d'abstractions (Wunenburger). Gaston Bachelard, qui s'inscrit dans cette perspective épistémologique, place l'image du côté de la poésie et l'oppose à l'intelligence scientifique (Bachelard 13). Or, dans de nombreuses œuvres produites à SymbioticA, l'imaginaire contamine l'objectivité de l'imagerie médicale. Il en est ainsi dans Structuring Somnolence (2010), œuvre participative de Lisa Carrie Goldberg, présentée à la Science Gallery de Dublin. Dans cette installation et 
performance, née d'une collaboration avec des spécialistes du sommeil, l'artiste revisite l'iconographie classique de l'endormi(e). Trois volontaires ont choisi des photographies de paysage ou d'architecture puis leur sommeil a été contrôlé de manière à ce que les courbes enregistrées imitent les contours des bâtiments ou du paysage choisis. Recréée dans une galerie ouverte sur la rue, cette expérience/performance associait la froideur blanche d'une chambre d'hôpital et les graphiques dépouillés des enregistrements à des images poétiques, celles des paysages et celles de l'endormi(e). La relation entre la technique et l'humain est ici reconsidérée : l'outil technique est au service non pas de la vérité scientifique mais de l'imagination. L'objectivité de l'instrument scientifique est remise en cause ; l'imagerie cède la place à l'imaginaire. Les transcriptions des phases du sommeil, transformées en œuvres graphiques, semblent être le produit des rêves des personnes alitées'.

La réintroduction de l'imaginaire opérée par les artistes traduit fréquemment les craintes que font surgir les recherches en biologie. Cela est particulièrement vrai lorsqu'elles nécessitent des expérimentations sur le vivant.

\section{De la mutation biologique au chimérique}

12 Les artistes en résidence font l'expérience d'une rencontre avec la vie et la mort. L'un des objectifs de SymbioticA est d'explorer les frontières, de plus en plus poreuses en raison des développements scientifiques, entre le non-vivant et le vivant ${ }^{10}$. De plus, la rencontre avec d'autres formes de vie (animale, microbienne, bactérienne, végétale) permet de prendre conscience de la continuité du vivant. Travaillant sur la confrontation avec la vie animale, Denis Viennet évoque une forme de rencontre qui

survient dans l'événement qui est dévoilement de l'hétérogénéité inattendue que sécrète un hasard inouï bouleversant l'homogénéité attendue d'un vivant. Elle renouvelle en accueillant l'autre au sein d'une vie qui est toujours plus que la vie, autodépassement, voire surpassement, passage à des formes nouvelles, transformation vers autre chose, par le jeu de forces parfois invisibles, de forces particulières et particulaires (Viennet).

Nombreux sont les artistes qui, frappés par l'expérimentation animale, conçoivent des dispositifs qui rendent hommage aux animaux de laboratoire et remettent en cause la hiérarchie entre l'homme et l'animal dans une démarche anti-spéciste. Ils font leurs les propos de Zurr : "We have to be careful of human arrogance. We need to be posthumanist. For us, species is not important. » (Solon) Les écrits de la biologiste Donna J. Harraway, visant à remettre en cause la binarité qui sous-tend notre rapport au végétal et au monde animal, mais aussi la dichotomie naturel/artificiel ou homme/machine, ont également eu une influence considérable sur les bio-artistes.

La répulsion suscitée par l'expérimentation animale est illustrée, de manière pionnière, dans An Experiment on a Bird in an Air Pump (1768) de Joseph Wright of Derby : dans de larges vases en verre, abritant la vie mais recueillant aussi la mort, sont emprisonnés un oiseau agonisant, un poumon préservé dans du liquide et un volatile déplumé. Certains spectateurs s'effraient de la cruauté du dispositif. Évoquant certaines vanités, la toile aborde la question de la fragilité de la vie mais aussi le spécisme. Dans certaines œuvres produites à SymbioticA, l'incubateur en verre devient un cercueil, une urne cinéraire ou un reliquaire. Le maintien de la vie en milieu stérile et la préservation du corps mort se confondent. Dans After-Life, The Immortalization of Kira and Rama, Svenja 
Johni Kraz s'inspire de l'art égyptien pour dessiner un incubateur-reliquaire contenant plusieurs fragments de veau. Le design et la création de ces réceptacles obéissent à des contraintes biologiques fortes (stérilité, température, etc.), tout en revisitant l'art funéraire. Autre artiste en résidence, Kathy High décrit son œuvre, Burial Globes, comme un mémorial rendant hommage aux rats ayant été utilisés comme cobayes. Les globes funéraires en verre boursouflé, rappelant les vases d'expérimentation, sont destinés à recevoir les cendres de cinq rats transgéniques nommés et individualisés. Une autre œuvre de l'artiste, HLA-B27, présentant des gros plans photographiques de rats de laboratoire à qui on a injecté de l'ADN humain et évoquant des foetus humains, nous incite à penser la continuité biologique entre l'homme et l'animal. La résidence de l'artiste a donné lieu à un manifeste intitulé rat love ${ }^{11}$ nous invitant à développer de l'empathie pour ces créatures. Dans ces œuvres, l'animal, objet de laboratoire, est érigé au rang de sujet, sujet de souffrance, sujet de débat tandis que la différence entre eux et nous devient incertaine.

de l'homme ou de l'indifférenciation des espèces a longtemps été perceptible dans le trope de la chimère. Les chimères créées par les bio-artistes peuvent mêler végétal et humain ${ }^{12}$ ou bien animal et humain ${ }^{13}$. Dans Embracing Animal (2004-2006) ${ }^{14}$, une installation et performance, Kathy High met en scène plusieurs métamorphoses. Reconstitution d'un laboratoire, l'œuvre inclut des rats vivants que les spectateurs peuvent toucher, des dispositifs expérimentaux et des vidéos montrant l'artiste nue, affublée de masques animaux et entrant en contact physique intime avec des chiens ou des chats, ainsi qu'un homme se métamorphosant en loup ou en ours. La notion deleuzienne de devenir-animal, centrée sur l'acceptation de l'altérité, sous-tend cette œuvre comme celles de Kira O'Reilly, autre artiste en résidence à SymbioticA. Créés au sein de l'institution scientifique, ces projets rappellent que "l'humain qui programme, organise et ordonne est traversé par de l'immaîtrisable, par un chaos qui le dépasse » (Viennet), un chaos animal interne à l'humain. "Nous sommes tous des bêtes, et des bêtes de laboratoire ", proclame Jean Baudrillard (191).

De telles affirmations sont mises à l'épreuve du corps dans le travail de Kira O'Reilly. En résidence à Perth, elle apprend la culture tissulaire à partir de cellules porcines avant d'envisager d'expérimenter la technique sur sa propre peau, une étape qui ne pourra être franchie. Cette culture a des vertus régénératrices qui stimulent l'imagination d'une artiste de longue date préoccupée par la cicatrice comme métaphore. Lors de sa résidence, elle a tenté de développer, en référence à la technique de la dentelle, la culture tissulaire sur une toile d'araignée à partir d'un prélèvement de son propre épiderme. Elle explique :

The spider silk tissue culture idea is an intriguing one, from both material, scientific and metaphorical points of view. (...) There is a significant and tantalising underlying strata of metaphorical and material interplays as both the spider and its silk unravel in and out of culture and mythologies. (O'Reilly)

17 Plusieurs photographies montrent l'épiderme de l'artiste enveloppé de toiles d'araignée et convoquent l'image d'une métamorphose arachnéenne. La peau y apparait comme un formidable organe sensoriel, une interface entre le corps interne, le corps externe et le monde extérieur. L'épiderme est aussi un élément majeur du système immunitaire et le contact avec la toile d'araignée évoque de possibles contaminations. Kira O'Reilly s'est de longue date intéressée à la peau et a réalisé des œuvres dans lesquelles elle se scarifiait ou déposait des sangsues sur son corps. Elle explore à la fois l'angoisse de la perte de ce que Didier Anzieu appelle le moi-peau ${ }^{15}$ et 
les possibles hybridations entre l'homme et l'animal. Les biotechnologies permettent en effet aux artistes contemporains d'aborder de manière très physique la question de l'instrumentalisation des animaux et des organismes vivants ainsi que celle de notre animalité.

Voici ce qu'Eduardo $\mathrm{Kac}^{16}$ rappelait, retraçant brièvement l'histoire de la chimère : « In their verbal, visual, or embodied forms, "monsters" have been used by society to project anxieties that reflect major cultural shifts taking place at different points in history. » (Kac 6) Pig Wings (2000-2001), œuvre réalisée par Oron Catts avec l'aide de l'ingénieur Guy Ben-Ary, évoque un corps monstrueux. L'artiste a prélevé des cellules porcines, utilisées pour traiter certaines pathologies cérébrales humaines, afin de les cultiver en forme d'ailes de manière à rendre admirable cet animal méprisé et jugé impur. Les trois ailes de formes différentes, génétiquement créées, ont été enduites d'or et cultivées pendant 9 mois. Les formes différentes de ces trois prothèses sont délibérées, Catts étant conscient que les créatures ailées peuvent être angéliques ou maléfiques. Bien que le résultat de l'expérience ne permette pas de contempler un cochon ailé, le projet pose la question de la manipulation du vivant et de la possibilité de réaliser l'imaginaire. La nouvelle plasticité du corps humain introduite par la génétique, les échanges inter-espèces induits par les xéno-transplantations, l'avènement de l'homme augmenté et du cyborg ravivent les angoisses liées aux limites fragiles de l'humain. Le chimérique, non plus comme image mais comme corps, témoigne des interrogations contemporaines sur une hybridité devenue tangible. Ionat Zurr note ainsi sa surprise face aux manipulations sur le vivant qu'il observe à SymbioticA et notamment à la greffe d'une oreille sur un rongeur: "It was such a strong image for artists-a surrealist's dream becoming a reality. We realized that life can be used as a raw material." (Solon) Comme le soulignent les directeurs de SymbioticA, la notion de corps et son usage changent au fur et à mesure que l'on acquiert de nouvelles connaissances et que l'on élabore de nouvelles technologies. Le corps n'est plus perçu comme un tout unifié mais comme un agencement de parties manipulables et remplaçables. La plasticité du corps devient réalité et la manipulation génétique, tout comme la création d'organismes à partir de cellules souches, fait écho à la plasticité des matériaux artistiques et à la création dans ses rapports à l'imagination. Le monstre (modification physique d'un être dont l'identité n'est pour autant pas hybride) et la chimère (mélangeant plusieurs espèces et remettant en cause l'identité corporelle (Andrieu 59)) continuent d'être perçus par l'homme comme une dégradation, une menace. Alors que d'autres artistes ont utilisé le motif du monstre ou de la chimère et les techniques de modification du corps (implants ou morphing) pour déconstruire les normes corporelles et l'appréhension de la déviance, contester l'indexation de l'art à la beauté ou interroger le rapport de l'organiste au mécanique, les bio-artistes revisitent le trope à l'aune de l'antispécisme : leurs œuvres contestent avant tout la taxonomie des organismes vivants et invitent à concevoir un corps organique étendu et non anthropocentré. Ils proposent de reconsidérer notre rapport à l'environnement naturel et aux organismes vivants que nos comportements mettent en danger, surtout à notre époque : " our well-established cultural dichotomies between self/other are shifting and the emphasis on differences are now being relocated in the continuum of life » (Catts et Zurr 2003, 170).

Shannon Bell, artiste canadienne féministe, performeuse et philosophe, aborde quant à elle dans ses œuvres la question de l'hybridité sexuelle. Lors de sa résidence à 
SymbioticA en 2005, Bell a utilisé les techniques de culture tissulaire pour produire un phallus féminin. Cette déclinaison de l'hermaphrodite interroge la construction sociale et culturelle de la féminité par opposition à la masculinité mais aussi à l'essentialisme. La réalisation d'un organe hermaphrodite réel modifie notre perception de la bisexualité en faisant basculer le fantasme dans une réalité biologique palpable. Two Phalluses and a Big Toe fait référence à une série de photographies surréalistes très suggestives de Jacques-André Boiffard montrant des gros orteils aux allures de phallus, et au texte de Bataille expliquant que le gros orteil représente ce qui est vil et déviant ${ }^{17}$. Comme chez Bataille, les deux sexes et le gros orteil recréés par Shannon Bell substituent au fétichisme poétique freudien une matérialité vile, une forme d'antiidéalisme. Dans la perspective féministe qui est celle de l'artiste, le phallus féminin créé nie l'idée de l'absence de phallus chez la femme et la perception du vagin comme béance dans de nombreuses théories psychanalytiques. Le phallus féminin a été créé à partir d'un moulage du clitoris de l'artiste, ensuite réduit puis imprimé en 3D pour réaliser un moule en silicone et une structure biodégradable en polymères servant de tuteur pour la culture cellulaire faite dans un bioréacteur. Les trois organes obtenus l'ont été à partir de cellules HeLa, une lignée cellulaire cancéreuse, collectées après un prélèvement de métastases effectué sur une patiente atteinte d'un cancer du col de l'utérus et décédée en 1951, Henrietta Lacks, une afro-américaine de Baltimore. Réunissant artistes et philosophes (Stellarc, Orland, et Bell), le projet Two Phalluses and a Big Toe prolonge l'idée de Heidegger selon laquelle l'art permet de révéler des entités nouvelles et une vérité cachée. La nature expérimentale du projet a rendu saillante la hantise de la mutation génétique. En effet, les rotations du bioréacteur ont provoqué le rapprochement des trois organes si bien que les deux phallus et le gros orteil ont fusionné en un nouvel organe inconnu. Les nouveaux organismes n'ont pas été durablement vivants mais leur évolution est documentée par une série de photographies montrant le processus jusqu'à l'extinction des organismes ${ }^{18}$. Dans les divers projets expérimentaux mentionnés, l'œuvre n'est plus constituée uniquement d'un objet, fût-il vivant, mais inclut les différentes étapes de l'expérimentation.

\section{Du protocole au rituel}

Le bio-art peut être appréhendé comme un prolongement de la démarche conceptuelle des artistes des années 1970, en ce qu'il consiste en l'élaboration de dispositifs, démarches et processus (Michaud 2003, 50). Le régime mimétique aurait laissé place à " un idéal du procédural ou idéal du protocolaire » (Joseph Mouton dans During, 152). Les artistes en résidence à SymbioticA élaborent une démarche artistique rigoureuse, adossée à des hypothèses scientifiques et leurs expérimentations, consignées dans des blogs par exemple ou dans ce que Laurent Jeanpierre appelle métaphoriquement des " boîtes noires" (Jeanpierre 310), font partie de l'œuvre. Cette dernière n'est pas le résultat d'une propédeutique qui resterait masquée mais constitue un projet dans son ensemble. Kira O'Reilly évoque :

A series of experiments (...) made with varying results, many of them generating compelling and intriguing dialogues across and between our respective disciplines. These interdisciplinary conversations were clearly as much a part of the work as the actual material processes. (O'Reilly)

21 La création est expérimentale : l'irruption de la matière vivante, dans la science comme dans l'art, introduit une indétermination (Zerbib 45, 51). L'expérimentation scientifique 
et artistique induit «étonnement, tâtonnement, doute, bricolage, erreur et ratage, etc. » (During 16). La pratique expérimentale et la possibilité de l'échec, artistique et scientifique, ébranlent la toute-puissance du chercheur comme celle de l'artiste, et s'inscrit plus largement dans la remise en question postmoderne de l'autorité de l'auteur (Zerbib 37). L'expérimentation artistique contemporaine se juge donc d'abord à son processus (Jeanpierre 312). Sa dimension performative est ainsi essentielle : du protocole scientifique au processus, de la manipulation à la gestuelle rituelle, le bio-art mobilise les corps de manière performative et symbolique.

Les artistes pratiquant la performance sont particulièrement sensibles à la scénographie du laboratoire, un lieu très sémiotisé, théâtre d'un rituel scientifique. La théâtralisation ou spectacularisation de la science, déjà mise en image par Joseph Wright of Derby, repose sur la mise en scène du corps (Desrochers 77). Jennifer Willet note son étonnement devant les technologies, les pratiques sociales et les règles du laboratoire ${ }^{19}$. Elle réalisa, avec Kira O'Reilly, une performance intitulée Be-wildering (2004) lors de laquelle, soit nues, soit en costumes blancs évoquant les combinaisons scientifiques, elles adoptaient des positions incongrues représentant la structure des chromosomes ou de l'ADN. La référence aux formes du mime ou de la pantomime, ainsi que le rideau blanc tendu dans l'embrasure du cénacle scientifique, soulignaient la théâtralité du laboratoire. En rendant étranges les symboles scientifiques (be-wildering suggère un étonnement et un être étrange) et en incarnant les maillons d'une chaîne d'ADN, elles rendaient visible le lien qui unit notre corps externe à notre patrimoine génétique invisible à l'œil nu.

Biologistes et artistes accomplissent des gestes répétés et minutieux, et manipulent des matières. Leurs pratiques font écho aux pratiques artisanales souvent évoquées d'ailleurs dans les œuvres (gravure, estampage, sculpture ou travaux d'aiguille). On soulignera les homologies entre tissu organique et trame textile, entre suture et couture, culture cellulaire et sculpture tissulaire. Elles sont au cœur de l'œuvre que l'artiste canadienne Tagny Duff réalisa à SymbioticA en 2012 avec l'aide de l'ingénieur David St Onge. Résultats de recherches en virologie et de techniques complexes, les objets exposés sont des livres en peau humaine et animale cryogénisés et présentés dans des bibliothèques portatives aux propriétés spécifiques. Dans ses commentaires sur l'œuvre, Duff insiste sur la manipulation de la matière :

Most of my time working in the lab is spent improving my pipetting technique. The more precise and agile I can be with pipetting, the cleaner, precise the measurement is, and the more likely my cells will proliferate and grow. ${ }^{20}$

L'artiste a mêlé des prélèvements de peau humaine à des cellules animales pour réaliser des livres semi-vivants à partir de la technique de la reliure qui évoque celle de la suture chirurgicale. La question de l'archivage est doublement déclinée : d'une part, la cryogénisation (un procédé de cryoconservation de tout ou de parties d'êtres vivants ou de cellules) est associée à la préservation et fait naître l'espoir de l'immortalité ; d'autre part, la conservation des livres dans des boîtes maintenant une température de - $80^{\circ} \mathrm{C}$ évoque l'image de la bibliothèque comme lieu dépositaire de toute culture. Technologie de pointe et formes anciennes se côtoient: la forme de la boîte-vitrine rappelle celle des bibliothèques portatives du $18^{\mathrm{e}}$ siècle permettant l'acheminement de livres dans les comptoirs ou postes reculés de la région des Grands Lacs en Amérique du Nord; l'artiste s'est inspirée des couvertures en peau humaine de manuscrits anciens conservés à la Welcome Trust Library, notamment le De integritatis \& corruptionis virginum notis (1663); elle a réalisé des tatouages viraux sur les couvertures, geste 
mêlant à nouveau haute technicité et coutumes anciennes. Corps et corpus sont réunis dans cette œuvre riche et complexe. Alors que le livre papier, après avoir été parchemin, est menacé de disparition, l'artiste recorporalise le livre, le fait à nouveau corpus. Le projet met en œuvre des protocoles divers qui deviennent rituels. Les gestes de l'artiste, répétés et techniques, sont ritualisés. Reposant sur une gestuelle répétée et codifiée, le rituel profane revêt des dimensions expressive, symbolique et performative illustrées dans l'œuvre de Tagny Duff comme dans d'autres œuvres produites à SymbiotcA. Comme tout rituel, le projet met en scène des objets symboliques, se déroule dans un lieu également investi d'une symbolique forte et face à des participants. L'espace social qui abrite le rituel en est la condition. L'interactivité caractérisant fréquemment le bio-art est générée par le rituel : les spectateurs sont invités à manipuler, toucher, alimenter, ou sacrifier les organismes vivants. Munis de gants, les visiteurs peuvent toucher les livres cryogénisés créés par Tagny Duff. L'hygiène relève du protocole scientifique mais aussi de l'histoire culturelle et sociale. De la même manière, les notions de contamination, de purification ou de vaccination, souvent mobilisées dans les œuvres, ont des résonances métaphoriques dans le domaine politique.

Dans plusieurs projets se pose la question du maintien en vie des organismes vivants ou semi-vivants exposés. Dans Victimless Leather, Oron Catts a tenté de faire se développer des cellules en forme de veste à partir d'une structure biodégradable en polymères. L'artiste a utilisé du sérum de veau fotal, SVF, communément utilisé en culture cellulaire, et des cellules souches embryonnaires de souris. L'œuvre est constituée de plusieurs étapes : la phase expérimentale de manipulation de cellules et d'organismes vivants; la sculpture des organismes grâce à des tuteurs; la documentation par une vidéo de la croissance des organismes ; la création d'incubateurs en vue de l'exposition ; l'exposition et l'alimentation quotidienne des organismes qui participe d'une éthique et d'une esthétique du soin ou de la sollicitude (care); enfin, le rituel de mise à mort de l'organisme. La veste organique s'est développée pendant 5 semaines puis s'est décomposée. En effet, le contact d'un organisme avec le monde extérieur ou la main d'un spectateur peut provoquer sa mort par contamination. Conscients de ce problème, les artistes travaillant avec des biomatériaux ont élaboré des rituels de mise à mort rappelant les rites sacrificiels, qui impliquent le conservateur ou directeur de la galerie et les spectateurs. Ce rituel pose la question éthique de la souffrance infligée et de la responsabilité individuelle face au soin exigé par certaines formes vivantes. $\mathrm{Si}$, de l'aveu des anthropologues, le rituel a une valeur régulatrice et renforce la cohésion sociale, les gestes de soin et de mise à mort effectués par les artistes et les spectateurs mettent en exergue la vulnérabilité partagée qui s'oppose à l'arrogance humaine que la recherche scientifique peut trahir. Parallèlement à l'invisibilité sociale théorisée par Guillaume Le Blanc, il y aurait une invisibilité biologique à laquelle le bio-art tenterait de remédier: en mettant en scène la vie et la mort, le bio-art rend tangible un continuum biologique qui étend la notion de communauté à l'ensemble du monde vivant.

\section{Conclusion}

Dans un ouvrage dont le propos est de démontrer que l'art est aussi vital que la science, Sîan Ede affirme que les capacités à visualiser, abstraire, imaginer, prétendre, raconter, 
représenter et réinterpréter les choses à l'infini, capacités qui sont au cœur de l'art, résultent de qualités humaines aussi essentielles que le discours rationnel et la présentation de preuves empiriques (Ede 155). Dans les projets qui ont vu le jour à SymbioticA, cette binarité est remplacée par une fécondité symbiotique qui naît de la rencontre entre le signe et l'image, l'imagerie et l'imaginaire, l'hybridité biologique et le chimérique, le protocole et le rituel. En replaçant le corps organique, la matière et la sensation au cœur de la démarche créatrice et au centre de l'appréhension des problèmes éthiques posés par la science, en élaborant des imaginaires pragmatiques résultant d'une heuristique ouverte dans laquelle l'imagination reste centrale, les tandems d'artistes et biologistes font œuvre d'envisionneurs. Le bio-art fait naître, dans les plis multiples de la matière qu'il explore des moments de suspens qui sont le propre de la pensée expérimentale. L'artiste Dominique Figarella rappelle que

si l'on considère l'expérience que l'on a d'un matériau avec une attention et une concentration suffisamment soutenues pour pouvoir traverser son usage consensuel, alors dans ce suspens, notre sensibilité produira immanquablement dans la sensation qui l'appréhende d'habitude, des ressemblances nouvelles, des points de contact pour des possibilités de liaisons qu'on n'aurait pu prévoir (Figarella 171).

Moment de suspens, le bio-art est alors davantage une involution qu'une évolution : « involuer, c'est former un bloc qui file suivant sa propre ligne, 'entre' les termes mis en jeu », notent Deleuze et Guattari (292).

\section{BIBLIOGRAPHIE}

Aloï, Giovanni. « The Death of the Animal ». Antennae, n 5, 2008, p. 43-53.

Andrieu, Bernard. « Embodying the Chimera: Biotechnology and Subjectivity ». In Signs of Life. Bio Art and Beyond. Edouardo Kac (dir.). Londres et Cambridge (Mass.) : The MIT Press, 2007. 57-68.

Bachelard, Gaston. La Formation de l'esprit scientifique, Paris : Vrin, 1967.

Baker, Steve. The Postmodern Animal. Londres : Reaktion Books, 2000.

---• « Contemporary Art And Animal Rights ». In Considering Animals: Contemporary Studies In Human-Animal Relations, Londres : Ashgate, 2002.

Bataille, Georges. « Le Gros orteil ». Documents n 6, Paris, 1929.

Baudrillard, Jean. « Les bêtes. Territoire et métamorphoses ». In Simulacres et simulation. Paris : Galilée, 1981.

Bouvais, Walter. « Le collectif SymbioticA expose son art du semi-vivant. De l'élevage de steaks de grenouille considéré comme un des beaux-arts ». 2003. http://www.transfert.net/a8577

(Consulté le 29 mai 2018).

Catts, Oron. « Why Artists Play with Life ». Bioart Special. Why Artists Play with Life, Mapping Bioart and DIY Bio, Biohackers are creators. Amsterdam : Waag Publication, Institute For Art, Science, And

Technology, 2012. 5-6. 
Catts, Oron, Zurr, Ionat. « Growing Semi-Living Sculptures ». Leonardo 35 : 4, Août 2002. 365-370.

---. « The Ethical Claims of Bioart: Killing the Other or Self-Cannibalism ». Aanz Journal of Art: Art And Ethics, $\mathrm{n}^{\circ} 4: 2,2003$ et $\mathrm{n}^{\circ} 5: 1,2004.167-188$.

Catts, Oron, Sillars Laura. « Artists and Scientists, a Symbiotic Relationship ? ». In Human Futures, Art In An Age Of Uncertainty. Andy Miah (dir.). Liverpool University Press \& Fact, 2008.

Daubner, Ernestine, Poissant, Louise (dir.). Bioart, Transformation Du Vivant. Montréal : Presses Universitaires du Québec, coll. Esthétique, 2012.

Deleuze, Gilles, Guattari, Félix. Mille Plateaux. Paris : Éditions de Minuit, 1980.

De Menezes, Marta. «The Artificial Natural: Manipulating Butterfly Wing Pattern for Artistic Purposes ». Leonardo, $\mathrm{n}^{\circ}$ 36. 1-29.

Desrochers, Christine. "Theâtralité, rituel et jeu dans le bioart ». In Pratiques performatives : body remix. Josette Féral (dir.). Rennes et Montréal : Presses Universitaires de Rennes et Presses Universitaires du Québec, 2012. 77-102.

Duff, Tagny. « Research in Brief, Cryobook Archive ». Canadian Journal Of Communication, $\mathrm{n}^{\circ}$ 37, 2012. 147-154.

Duggan, Patrick. «The Touch and the Cut: an Annotated Dialogue with Kira O'Reilly ». Studies in Theatre and Performance, $n^{\circ} 29: 3,2009$.

Dumas, Stéphane. Les Peaux créatrices, esthétique de la sécrétion. Paris : Klincksieck, coll. Eshétique, 2014.

During, Elie et al. (dir.). In Actu. De l'expérimental dans l'art. Annecy : Les Presses du Réel, 2009.

Ede, Sîan. Art \& science. New York : I.B. Tauris, 2005.

Figarella, Dominique. «Conduire sans permis ». In In Actu. De l'expérimental dans l'art. During, Elie et al. (dir.). Annecy : Les Presses du Réel, 2009. 159-173.

Francis, Michelle (dir.). Adaptation. Perth : University of West Australia, 2012.

Gigliotti, Carol. « Heartburn: Indigestion, Contention and Animals in Contemporary Art ». Antennae 14 : The Politics Of Meat, Automne 2010. 25-33.

Grosz, Elizabeth. Volatile Bodies: Towards a Corporeal Feminism. Crows Nest, NSW: Allen \& Unwin, 1994.

Harraway, Donna J. When Species Meet (Posthumanities). Minneapolis : University of Minnesota Press, 2007.

Hauser, Jens. « Derrière l'animal l'homme ? Altérité et parenté dans l'art biotech ». Animaux d'artistes : Figures de l'art n 8. Bernard Lafargue (dir.). 2003-2004. 397-426.

-- - (dir.). Sk-interfaces: Exploding Borders-Creating Membranes in Art, Technology and Society. Liverpool, FACT : Liverpool University Press, 2008.

Jeangène Vilmer, Jean Baptiste. « Animaux dans l'art contemporain : la question éthique ». Jeu : Revue de Théâtre, $\mathrm{n}^{\circ} 130: 1,2009$. 40-47.

Jeanpierre, Laurent. "Introduction aux conditions de l'art expérimental », in Elie During et al. (ed.), In Actu, De l'Expérimental dans l'art, Annecy, Les Presses du Réel, 2009, p. 307-335.

Kac, Eduardo (dir.). Signs of Life. Bio Art and Beyond. Londres et Cambridge (Mass.) : The MIT Press, 2007. 
Kelley, Jeff (dir.). Kaprow Allan, Essays on the Blurring of Art and Life. Berkeley et Los Angeles : University of California Press, 2003.

Le Blanc, Guillaume. L'Invisibilité sociale. Paris : Presses Universitaires de France, 2009.

Michaud, Yves. L'Art à l'état gazeux, essai sur le triomphe de l'esthétique. Paris : Stock, 2003.

---. « Arts et biotechnologies ». L'art Bio-Tech. Nantes : Le Lieu Unique, 2003. 80-85.

---. Humain, inhumain, trop humain. Paris : Climats, 2006.

O’Reilly, Kira. « Notes from the Lab ». http://www.interact.mmu.ac.uk/placements/documents/ 30/Notes\%20from\%20the\%20lab.pdf (Consulté le 29 mai 2018).

Poissant, Louise. « Arts et Sciences, les biotechnologies et le bioart ». Bioart, Transformation du vivant. Ernestine Daubner et Louise Poissant (dir.). Montréal : Presses Universitaires du Québec, coll. esthétique, 2012. 15-36.

Sawchuck, Kim. « Hypermasculinity, Ageing Bodies and Fast Feminisms: An Interview with Shannon Bell ». http://nomorepotlucks.org/site/hypermasculinity-ageing-bodies-and-fastfeminisms-an-interview-with-shannon-bell/ (Consulté le 25 mai 2018).

Serres, Alexandre. «Quelle(s) problématique(s) de la trace?». Texte d'une communication prononcée lors du séminaire du CERCOR (actuellement CERSIC), 13 déc. 2002. https:// archivesic.ccsd.cnrs.fr/sic 00001397.

Solon, Olivia. « Bioart: the Ethics and Aesthetics of Using Living Tissue as a Medium ». http:// www.wired.com/2011/07/bioart/ (Consulté le 29 mai 2018).

Stracey, Frances. « Bio-Art: the Ethics behind the Aesthetics ». Perspectives n 10, 2009. 498. http://www.nature.com/nrm/journal/v10/n7/abs/nrm2699.html (Consulté le 29 mai 2018).

Thorens, Adèle. « Peter Singer et la libération animale : une approche critique autour de la notion de spécisme ». Revue de théologie et de philosophie, $\mathrm{n}^{\circ} 134: 2,2004$. 149-165.

Viennet, Denis. « Animal, animalité, devenir-animal ». Le Portique, n²3-24, 2009. https:// leportique.revues.org/2454?lang=en (Consulté le 29 mai 2018).

Voison, Catherine. «Les animaux d'artistes au regard des pratiques de laboratoire ». Que la bête meure! L'animal et l'art contemporain. Marion Duquerroy (dir.). Université Paris 1, 2012. http:// hicsa.univ-paris1.fr/page.php?r=133\&id=693\&lang=fr (Consulté le 29 mai 2018).

---. « L'art contemporain au prisme des biotechnologies ». Amnis, n 13, 2014. http:// amnis.revues.org/2171 (Consulté le 29 mai 2018).

Wallace, Carmel. «Lake Life: a Micro Approach to Art and Ecology ». Imprint, n 48 : 2, 2013. 14.

Watt, Yvette. « Artists, Animals, and Ethics ». Antennae 19, Animal Wrongs And Rights. Hiver 2011. 62-73.

Willet, Jennifer. « Performing Biotechnology: Reimagining Inter-Artist/Interspecies Interrelations in the Laboratory with Kira O'reilly ». E-Misférica, Bio/Zoo, nº $10: 1$, 2013. http:// hemisphericinstitute.org/hemi/en/e-misferica-101/willet (Consulté le 29 mai 2018).

---. « Bodies in Biotechnology: Embodied Models for Understanding Biotechnology in Contemporary Art ». Leonardo Electronic Almanac, n 7-8, 14, 2006.

---. « Creators - Jennifer Willet ». https://artthescience.com/blog/2017/09/28/creators-jenniferwillet/ (Consulté le 29 mai 2018). 
Wunenburger, Jean-Jacques. « Métaphore, Poïétique et Pensée Scientifique ». Revue européenne des sciences sociales. XXXVIII-117, 2000. http://ress.revues.org/707. (Consulté le 29 mai 2018).

Zerbib, David. «Les noms du per : l'art expérimental et l'épreuve des limites », in Elie During et al. (ed.), In Actu, De L'expérimental dans L'art, Annecy, Les Presses Du Réel, 2009, p. 33-56.

Zwinjenberg, Robert. « Bioart : Performance in Transformation ». Bioart Special. Amsterdam : Waag Society Publication, 2012.

\section{NOTES}

1. Voir Oron et Zurr, 2003, 168.

2. «Do artists cross a line when they breed plants or animals, or use the tools of biotechnology? Scientists routinely cross the line. (...) Only artists and certain religious people hesitate. Of course, one of the great human dilemmas is that we do not know the extent of our powers. We invent outrageously and as casually as we breathe, but we have no idea where our inventions will take us. (...) To the extent that art favours awareness, the more artists who cross the line the better. » (Oron et Zurr 2003, 167). Ionat Zurr et Catts n'occultent pas la tension existant entre la volonté des bio-artistes de rompre avec le spécisme et l'utilisation de leur position privilégiée d'humains et d'artistes pour pratiquer des expériences techniques et artistiques sur des animaux (Oron et Catts 2003, 184).

3. Voir Aloï, Baker, Zurr et Catts, Stracey, Gigliotti, Jeangène-Vilmer, Watt.

4. Dans son compte-rendu de L'Art bio-tech, exposition présentée à Nantes réunissant plusieurs artistes ayant été en résidence à Perth, Walter Bouvais explique que ces artistes « mènent leur art à la limite » et « portent au grand jour des scénarios réalisables, bien qu'extrêmes, parmi lesquels la culture de nos propres cellules hors de notre corps et à des fins alimentaires ». (Bouvais)

5. Allan Kaprow, « Experimental Art », 1966 (Kelley 69).

6. Dans Volatile Bodies: Towards a Corporeal Feminism, Elizabeth Grosz évoque la définition plurielle $\mathrm{du}$ corps : " a plural, multiple field of possible body "types," no one of which functions as the delegate or representative of the others, [which] must be created, a "field" of body types-young and old, black and white, male and female, animal and human, inanimate and animate-which, in being recognized in their specificity, cannot take the coercive role of singular norm or ideal for all the others. » (Grosz 22)

7. https://archive.org/details/GLACK

8. Site web de l'artiste, http://carmelwallace.com/symbiotica/

9. Dans le cadre du même projet, l'artiste a réalisé des portraits photographiques d'endormis équipés de volumineux masques médicaux et de tuyaux. Intitulée « The New Sleep ", cette série photographique évoque le contrôle du sommeil dans des sociétés totalitaires déterminées à contrôler ou restreindre le repos. Elle offre aussi une vision désenchantée du sommeil comme temps de liberté et de rêve.

10. Les fondateurs du laboratoire affirment: «We believe that the benefits to the viewers are that they will be drawn to reassess their perceptions of life in the light of their encounter with a real tangible example of the concept of partial life. This will hopefully assist them in forming an informed opinion in regard to developments in the bio medical field, and will provide them with the opportunity to meditate on what it means to be alive. » (Catts et Zurr 2003, 184)

11. http://www.embracinganimal.com/ratlove.html

12. On citera l'exemple d'un pétunia génétiquement modifié contenant de l'ADN humain, créé par Edouardo Kac, ou du cactus chevelu de Laura Cinti (Cactus Project) exposé en 2013 à la Fondation EDF à Paris, le produit d'une transgénèse qui consiste à faire fusionner un gène humain, celui de l'artiste, avec le génome d'une plante. 
13. L'exemple le plus frappant est Que le cheval vive en moi, une xénotransfusion réalisée lors d'une performance en 2011, dans une galerie de Ljubljana, par Marion Laval Jeantet, assistée de Benoît Mangin, qui a consisté à implanter dans l'organisme de l'artiste du sérum issu d'un cheval destiné à l'abattoir.

14. Voir http://kathyhigh.com/project-embracing-animal.html

15. Didier Anzieu explique que le sentiment d'inquiétante étrangeté peut être produit par un autre indiscernable à l'intérieur des enveloppes du moi et qui fait surgir la menace d'une perte des limites, de la contenance et de l'individualité (Dumas 80).

16. L'art génétique d'Eduardo Kac fut séminal même si tous les bio-artistes ne se tournent pas vers l'art transgénique dont il fut le pionnier. Sur ces premiers développements de l'art génétique, voir Daubnier 1-15.

17. Georges Bataille, « Le Gros orteil ».

18. Voir l'interview de l'artiste par Kim Sawchuck.

19. «I was amazed by the technologies, the social practices, and rules and regulations of the lab. » https://artthescience.com/blog/2017/09/28/creators-jennifer-willet/

20. L'approche expérimentale de l'artiste apparaît dans les textes de son blog, décrivant ses différentes démarches. http://cryobookarchives.wordpress.com/category/development/

\section{RÉSUMÉS}

Loin de servir la démocratisation des savoirs scientifiques, les pratiques des artistes en résidence à SymbioticA (Perth, Australie) utilisent le laboratoire comme atelier et les matières organiques comme matériaux, créant au terme de processus expérimentaux longs des reconfigurations artistiques. Nourrissant les débats éthiques autour de l'expérimentation animale, leurs œuvres transgressent les règles esthétiques et transfèrent le paradigme expérimental au domaine de la création artistique. Au cœur de ces œuvres-processus se trouvent l'imprévisibilité et l'hybridation qui remettent en question la définition de l'œuvre comme objet clos. Les intersections entre science et art sont multiples, depuis la dérive de l'imagerie vers l'image et l'imaginaire jusqu'à la déclinaison artistique et biologique de la composition. Les collaborations ouvrent sur une phénoménologie artistique où geste ritualisé et manipulations stimulent l'imaginaire et où les techniques scientifiques croisent le savoir artisanal. Elles aboutissent aussi à la création de chimères et à une réflexion sur les notions de care et de post-humain.

Far from merely democratizing scientific research, the artists in residency at SymbioticA (Perth, Australia) use the laboratory as a studio or workshop and organic substances as artistic material. The long experimental processes that they carry out lead to artistic reconfigurations. Besides fuelling the ethical debates over animal experimentation, their works transgress aesthetic codes and transfer the experimental paradigm to the field of artistic creation. Essential to their projects, challenging the idea of a finite artwork, are the notions of unpredictability and hybridity. The intersections between science and art are multifarious, ranging from the drift from imaging to image and to the imaginary, to the overlapping artistic and biological perceptions of composition. The collaborations open up onto an artistic phenomenology in which gesture and manipulation foster images and metaphors while scientific techniques blend with craftsmanship. They allow a materialisation of the chimera triggering considerations on the notions of care and the posthuman. 
INDEX

Mots-clés : Bio-art, résidence d'artiste, motif, chimère, spécisme, culture tissulaire, tissu, hybridation, animalité, rituel

Keywords : Bio-art, artistic residency, pattern, chimera, specism, tissue culture, textile, hybridation, animality, ritual

\section{AUTEUR}

\section{VALÉRIE MORISSON}

Maître de conférences

Université Bourgogne Franche-Comté, Textes Images Langages, EA 4182

valerie.morisson@u-bourgogne.fr 